\title{
A genome-wide scan for candidate lethal variants in Thoroughbred horses.
}

Evelyn T. Todd ${ }^{*}$, Peter C. Thomson ${ }^{1}$, Natasha A. Hamilton², Rachel A. Ang ${ }^{1}$, Gabriella Lindgren $^{3,4}$, Åsa Viklund ${ }^{3}$, Susanne Eriksson ${ }^{3}$, Sofia Mikko ${ }^{3}$, Eric Strand ${ }^{5}$ and Brandon D. Velie ${ }^{1}$.

${ }^{1}$ School of Life and Environmental Sciences, The University of Sydney, NSW 2006, Australia.

${ }^{2}$ Racing Australia Equine Genetics Research Centre, Racing Australia, NSW 2000, Australia.

${ }^{3}$ Department of Animal Breeding and Genetics, Swedish University of Agricultural Sciences, Uppsala, Sweden.

${ }^{4}$ Livestock Genetics, Department of Biosystems, KU Leuven, Leuven, Belgium.

${ }^{5}$ Department of Companion Animal Clinical Sciences, Faculty of Veterinary Medicine, Norwegian University of Life Sciences, Oslo, Norway.

*Correspondence and requests for materials should be addressed to Evelyn T. Todd (email: evelyn.todd@sydney.edu.au) 
2 Recessive lethal variants often segregate at low frequencies in animal populations, such that two randomly selected individuals are unlikely to carry the same mutation. However, the likelihood

4 of an individual inheriting two copies of a recessive lethal mutation is dramatically increased by

5 inbreeding events. Such occurrences are particularly common in domestic animal populations,

6 which are often characterised by high rates of inbreeding and low effective population sizes. To

7 date there have been no published investigations into the presence of specific variants at high

8 frequencies in domestic horse populations. This study aimed to identify potential recessive lethal

9 haplotypes in the Thoroughbred horse breed, a closed population that has been selectively bred

10 for racing performance.

11 In this study, we scanned genotype data from Thoroughbred horses $(n=526)$ for adjacent single

12 nucleotide polymorphisms (SNPs) at high heterozygote frequencies, but with a complete absence

13 of homozygotes. Two SNPs that matched these criteria were mapped to an intronic region in the

$14 L Y 49 B$ gene, indicating that a closely linked mutation may cause lethality in homozygous state.

15 Despite a complete absence of homozygotes, almost $35 \%$ of Thoroughbreds included in these analyses were heterozygous for both SNPs. A similar loss or absence of homozygotes was

17 observed in genotype data from other domestic horse breeds $(n=2030)$. Variant analysis of

18 whole-genome sequence data $(n=90)$ identified two SNPs in the $3^{\prime} \mathrm{UTR}$ region of the $L Y 49 B$

19 gene that may result in loss of function. Analysis of transcriptomic data from equine embryonic

20 tissue revealed that $L Y 49 B$ is expressed in the trophoblast during placentation stage of

21 development. 
22 In this study, a region in the $L Y 49 B$ gene was identified as a strong candidate for harbouring a

23 variant causing lethality in homozygous state. These findings suggest that $L Y 49 B$ may have an

24 essential, but as yet unknown function in the implantation stage of equine development. Further

25 investigation of this region may allow for the development of a genetic test to improve fertility rates in horse populations. Identification of other lethal variants could assist in improving natural

27 levels of fertility in horse populations.

\section{Author Summary}

Recessive lethal mutations may reach high frequencies in livestock populations due to selective breeding practices, resulting in reduced fertility rates. In this study, we characterise recessive lethal mutations at high frequencies in the Thoroughbred horse population, a breed with high rates of inbreeding and low genetic diversity. We identified a haplotype in the $L Y 49 B$ gene that shows strong evidence of being homozygous lethal, despite having high frequencies of

34 heterozygotes in Thoroughbreds and other domestic horse breeds. Two 3'UTR variants were

35 identified as most likely to cause loss of function in the $L Y 49 B$ gene, resulting in lethality. This

36 finding provides novel insights into the potential importance of $L Y 49 B$ in equine development.

37 Additionally, this study may assist with breeding strategies to improve fertility rates in the

38 Thoroughbred and other domestic horse breeds.

Introduction

There is estimated to be a high rate of natural embryonic mortality in mammals. A large

41 proportion of these embryonic losses occur soon after fertilisation, such that pregnancies often go

42 undetected, with the only sign being reduced fertility (1). Mutation screens in mice reveal that

43 many genes are essential for development, with knockout of $29 \%$ of genes tested resulting in 
44 embryonic death by day $14(2,3)$. Although mutations in these genes are expected to be under

45 strong negative selection due to being completely deleterious, many species are estimated to

carry between one and two recessive lethal mutations per genome (4). However, single mutations are often uncommon in a population, such that unrelated individuals are unlikely to carry the same recessive lethal mutations (5-7).

The likelihood of an individual inheriting two copies of the same lethal mutation is dramatically increased by inbreeding events, whereby alleles that are identical by descent are inherited from a common ancestor (8-10). Populations with high levels of inbreeding and low effective population size $\left(N_{e}\right)$ are at risk of these otherwise rare lethal mutations drifting to high frequencies. Endangered and fragmented species are particularly susceptible due to their small population size and low genetic diversity (11). Recessive lethal mutations can also been found at high frequencies in domestic animal populations despite their large census size due to inbreeding for the selection of desirable traits (12).

In recent years, a number of studies in livestock have identified embryonic lethal mutations at high frequencies due to intensive selective breeding practices (13-20). This is often due to a limited number of sires with desirable characteristics making large genetic contributions to the population $(13,21)$. Moreover, population bottlenecks due to domestication and breed formation have also resulted in increased deleterious mutation loads and diminished gene pools in many domestic breeds $(12,22-24)$. These processes lead to genetic variation and $N_{e}$ being reduced in future generations, which increases the chance of drift and inbreeding events. Lethal mutations that have reached high frequencies are often detected by deviations from the HardyWeinberg equilibrium with a lack of homozygotes for one allele (14). Characterisation of such 
mutations can assist in improving breeding decisions to increase fertility rates in these populations and prevent these mutations from drifting to higher frequencies $(25,26)$. horse populations. Although a recent study has identified some candidate mutations (27), to date there has been no published comprehensive characterisation of common embryonic lethal alleles in horse populations. Despite the large variety of domestic horse breeds found throughout the world, many breeds suffer from low within-breed diversity and small $N_{e}(28-30)$. Genetic variation in most horse breeds has decreased markedly within the last 200 years, largely due to their replacement by machinery in agriculture and transport $(31,32)$. Some horse breeds with large consensus population sizes also experience low $N_{e}$ and genetic diversity due to intense artificial selective breeding practices and closed population structures (29, 30). Maintaining good

77 fertility rates is particularly important for horse populations due to the seasonal nature of breeding and the low individual fertility output, as mares produce only one foal from an eleven month gestation period (33). Despite the extensive use of hormonal therapies to increase covering success in many domestic horse populations, per cycle pregnancy rates in some breeds

81 only average around $65 \%$, suggesting the presence of unknown variables that may reduce 82 fertility (34). In this study, we aimed to characterise variants at high frequencies that may cause lethality in the Thoroughbred horse population. The Thoroughbred breed is of particular interest

85 due to the closed population structure since the foundation of the studbook in the $18^{\text {th }}$ century

86 (35). The population has since been intensely selected for the improvement of athletic abilities

$87(36,37)$, resulting in contemporary Thoroughbred horses being characterised by high levels of inbreeding and a small $N_{e}(30,38-40)$. Due to selective breeding practices, all Thoroughbred 
horses can trace their ancestry back to a small number of individuals from the foundation of the breed $(38,39)$. Genetic diversity in the Thoroughbred breed has been reduced in recent decades due to the increased commercialisation of popular stallions providing large genetic contributions to the population (41). Although such practices are in line with selective breeding principles (42), they could also inadvertently increase the frequency of embryonic lethal variants in the population. Reproductive technologies such as artificial insemination are banned in the Thoroughbred population, making the maintenance of high levels of natural fertility imperative. Additionally, Thoroughbred horses have been used as foundation stock for other popular horse breeds including The Quarter Horse, Standardbred, and Warmblood (29), thus identification of embryonic lethal variants in Thoroughbreds is also likely to assist in the breeding management of these populations. Therefore, we also aimed to determine the frequency of any potentially lethal variants identified in the Thoroughbred population in other horse breeds and examine their transcriptomic profile in embryonic tissue.

\section{Results}


111 Thoroughbreds across both of these datasets were heterozygous for this two-SNP haplotype.

112 These SNPs also showed an absence or reduction of minor homozygotes in genotype data for

113 other domestic horse breeds ( $n=2030$, Table 2$)$.

These two candidate SNPs mapped to the coordinates of 6:38278097 and 6:38278874,

115 which are found in an intronic region of the $L Y 49 B$ gene on ECA6. This gene is part of the LY49

116 gene family, which plays an important role in innate immunity. There are five functional

117 members of the LY49 gene family in Equus caballus, all of which closely grouped together on

118 chromosome 6. Since both of the SNPs mapped to a non-coding region of the $L Y 49 B$ gene, the

119 likelihood of either being a causal variant for lethality is low.

Table 1: The allele frequencies of two adjacent SNPs with an absence of minor homozygotes in

122 genotype data from two Thoroughbred horse datasets. The expected number of minor

123 homozygotes in each population was calculated under Hardy-Weinberg equilibrium.

\begin{tabular}{|c|c|c|c|c|c|c|c|c|c|c|}
\hline \multirow[b]{2}{*}{ Population } & \multirow{2}{*}{$\begin{array}{l}\text { Sample } \\
\text { size }\end{array}$} & \multirow[b]{2}{*}{ Reference } & \multicolumn{4}{|c|}{ 6:38278097 } & \multicolumn{4}{|c|}{$6: 38278874$} \\
\hline & & & $\begin{array}{c}\text { Expected } \\
\text { GG }\end{array}$ & GG & AG & AA & $\begin{array}{l}\text { Expected } \\
\text { CC }\end{array}$ & CC & AC & AA \\
\hline $\begin{array}{l}\text { Australian } \\
\text { Thoroughbreds }\end{array}$ & 156 & Own data & 7 & 0 & 66 & 90 & 7 & 0 & 66 & 90 \\
\hline $\begin{array}{l}\text { Japanese } \\
\text { Thoroughbreds }\end{array}$ & 370 & $\begin{array}{l}\text { Fawcett et al., } \\
\text { 2019(43) }\end{array}$ & 9 & 0 & 117 & 253 & 9 & 0 & 117 & 253 \\
\hline
\end{tabular}


124 Table 2: Allele frequencies of SNPs in domestic horses at positions 6:38278097 and 6:38278874 on the EquCab2.0 assembly. The total number of horses included in this analysis was 2030.

\begin{tabular}{|c|c|c|c|c|c|c|c|c|c|c|}
\hline \multirow{2}{*}{ Breed } & \multirow{2}{*}{$\begin{array}{c}\text { Sample } \\
\text { size }\end{array}$} & \multirow{2}{*}{ Reference } & \multicolumn{4}{|c|}{ 6:38278097 } & \multicolumn{4}{|c|}{$6: 38278874$} \\
\hline & & & $\begin{array}{c}\text { Expected } \\
\text { GG }\end{array}$ & GG & AG & AA & $\begin{array}{c}\text { Expected } \\
\text { CC }\end{array}$ & CC & $A C$ & AA \\
\hline Swedish Warmblood & 380 & $\begin{array}{l}\text { Privately provided, } \\
\text { Ablondi et al., } \\
2019(44)\end{array}$ & 4 & 0 & 75 & 304 & 4 & 0 & 74 & 306 \\
\hline $\begin{array}{l}\text { Norwegian-Swedish } \\
\text { Coldblooded Trotter }\end{array}$ & 646 & $\begin{array}{l}\text { Privately provided, } \\
\text { Velie et al., } \\
2018(45)\end{array}$ & 26 & 0 & 258 & 388 & 28 & 22 & 226 & 393 \\
\hline Quarter Horse & 137 & $\begin{array}{l}\text { Petersen et al., } \\
2014(46)\end{array}$ & 17 & 0 & 97 & 40 & 17 & 0 & 97 & 40 \\
\hline Exmoor Pony & 285 & $\begin{array}{l}\text { Velie et al., } \\
2016(47)\end{array}$ & 0 & 0 & 1 & 279 & 0 & 0 & 1 & 282 \\
\hline Various breeds & 582 & $\begin{array}{l}\text { Petersen et al., } \\
2013(29)\end{array}$ & 15 & 0 & 85 & 497 & 15 & 0 & 85 & 497 \\
\hline
\end{tabular}


127

Phylogenetic origin of the candidate SNPS

According to the phylogenetic tree generated by Petersen et al $(28,29)$, and their associated SNP data, the SNPs of interest were present in heterozygous state across most phylogenetic branches of domestic horse breeds. Of the 32 breeds in this dataset, 23 had at least one heterozygote for both SNPs of interest. Notably, this two-SNP haplotype was not found in genotype data from one branch of the tree which contains the North Swedish Horse $(n=19)$, Norwegian Fjord Horse $(n=21)$ and Exmoor Pony $(n=24)$ (Table S1). A larger sample of Exmoor Pony data $(n=274$, Table 2$)$ found only one heterozygote for this haplotype.

Frequency of the candidate SNPs in other breeds

Analysis of SNP data from other domestic breeds showed that heterozygotes for the SNPs of interest were at a particularly high frequency in the Quarter Horse population $(71 \%, n=$ 137) (Table 2, Table S2). The proportion of heterozygotes was also high in Swedish Warmbloods $(n=380)$ and Norwegian-Swedish Coldblooded Trotters $(n=641)$, being $20 \%$ and $40 \%$ respectively (Table 2$)$. Smaller datasets also revealed that Belgian Draft $(n=19)$, French Trotter $(n=17)$, Paint $(n=15)$, Morgan $(n=19)$, Mongolian Paulista $(n=19)$ and Tuva $(n=15)$ breeds may also have a high proportion of heterozygotes for this haplotype in their populations (Table S1).

Identifying candidate causal variants using whole genome sequence data

To further investigate SNP frequencies in this region, variants were called from wholegenome sequence data of 90 domestic horses. The two SNPs identified in the preliminary analysis showed a complete absence of homozygotes for their minor alleles in these individuals (Table S3). Additionally, a number of variants closely linked to these SNPs were identified 
149 (Table 3, Figure S1). Annotation of these loci using SIFT(48) identified three variants that may 150 result in changes to protein structure or expression, so these represent the most likely candidates 151 to cause lethality in homozygous state (Figure 1).

The first of these variants, $6: 38282610 \mathrm{G}>\mathrm{A}$, was located in an exonic region of the $153 L Y 48 B$ gene and resulted in an amino acid change from a phenylalanine to a serine residue. This

154 SNP is located next a tryptophan residue that appears to be highly conserved across members of 155 the LY49 family and across species. However, there is little conservation of the phenylalanine 156 residue across taxa; some species have a phenylalanine and others a serine at this position. This 157 SNP is annotated as being "tolerated" in SIFT.

Two other variants that were closely linked to the candidate SNPs $(6: 38276742 \mathrm{~A}>\mathrm{T}$ and $6: 38276955 \mathrm{G}>\mathrm{A}$ ) were found within the 3'UTR region of the $L Y 49 B$ gene. Alignment of the

160 3'UTR region of the five functional LY49 genes in Equus caballus revealed that the region 161 containing the SNP 6:38276955G $>$ A is highly conserved in all members of the LY49 gene family

162 (Table 4). This region may be important for mRNA stability and translation into a functional 163 protein. The other variant was found in an AU-rich region at the end of the LY49B mRNA 164 transcript, which is often associated with polyadenylation and post translation stability. 
Table 3: Variants linked to the SNPs at 6:38278097 and 6:38278874 on the EquCab2.0

with a star.

\begin{tabular}{|c|c|c|c|c|}
\hline Variant & $r^{2}$ & $D^{\prime}$ & $\begin{array}{l}\text { Distance from } \\
\text { 6:38278097-6:38278874 }\end{array}$ & Annotation \\
\hline 6:38223012 & 0.83 & 1 & 55085 & Intronic Variant LY49F \\
\hline 6:38264682 & 0.81 & 1 & 13415 & Intronic Variant LY49F \\
\hline $6: 38265144$ & 0.85 & 0.92 & 12953 & Intronic Variant LY49F \\
\hline 6:38273491 & 0.87 & 1 & 4606 & Intronic Variant LY49F \\
\hline $6: 38273498$ & 0.87 & 1 & 4599 & Intronic Variant LY49F \\
\hline $6: 38273500$ & 0.87 & 1 & 4597 & Intronic Variant LY49F \\
\hline $6: 38273759$ & 0.93 & 1 & 4338 & Intronic Variant LY49F \\
\hline 6:38274367 & 1 & 1 & 3730 & Intronic Variant LY49F \\
\hline $6: 38274942$ & 1 & 1 & 3155 & Intronic Variant LY49F \\
\hline $6: 38276456$ & 1 & 1 & 1641 & Intronic Variant LY49F \\
\hline $6: 38276742 *$ & 0.83 & 1 & 1355 & 3'UTR variant LY49B \\
\hline $6: 38276955^{*}$ & 1 & 1 & 1142 & 3'UTR variant LY49B \\
\hline 6:38278097 & 1 & 1 & 0 & Intronic Variant LY49B \\
\hline $6: 38278874$ & 0.93 & 1 & 0 & Intronic Variant LY49B \\
\hline 6:38281733 & 1 & 1 & 2859 & Intronic Variant LY49B \\
\hline 6:38282610* & 1 & 1 & 3736 & Missense variant LY49B \\
\hline 6:38284541 & 0.93 & 1 & 5667 & Synonymous variant LY49B \\
\hline $6: 38285848$ & 0.85 & 0.92 & 6974 & Intronic Variant LY49B \\
\hline 6:38290614 & 0.85 & 0.92 & 11740 & Intergenic variant \\
\hline 6:38292923 & 0.86 & 0.93 & 14049 & Intergenic variant \\
\hline 6:38294589 & 0.85 & 0.92 & 15715 & Intergenic variant \\
\hline
\end{tabular}




\begin{tabular}{|l|r|r|r|l|}
\hline $6: 38296564$ & 0.85 & 0.92 & 17690 & Intergenic variant \\
\hline $6: 38297140$ & 0.86 & 0.93 & 18266 & Intronic Variant MAGOHB \\
\hline $6: 38297149$ & 0.86 & 0.93 & 18275 & Intronic Variant MAGOHB \\
\hline $6: 38304295$ & 0.83 & 0.91 & 25421 & Intronic Variant MAGOHB \\
\hline $6: 38319283$ & 0.81 & 0.9 & 40409 & Intergenic variant \\
\hline $6: 38334772$ & 0.82 & 0.91 & 55898 & Intergenic variant \\
\hline $6: 38341477$ & 0.86 & 0.93 & 62603 & Intergenic variant \\
\hline $6: 38341607$ & 0.86 & 0.92 & 62733 & Intergenic variant \\
\hline $6: 38345686$ & 0.86 & 0.93 & 66812 & Intergenic variant \\
\hline $6: 38349116$ & 0.86 & 1 & 70242 & Intronic Variant LY49C \\
\hline $6: 38349743$ & 0.85 & 1 & 70869 & Intronic Variant LY49C \\
\hline $6: 38351619$ & 0.8 & 0.93 & 72745 & Intronic Variant LY49C \\
\hline $6: 38352677$ & 0.89 & 1 & 73803 & Intronic Variant LY49C \\
\hline $6: 38353265$ & 0.85 & 1 & 74391 & Intronic Variant LY49C \\
\hline $6: 38354110$ & 0.86 & 0.93 & 75236 & Intronic Variant LY49C \\
\hline $6: 38358475$ & 0.83 & 1 & 79601 & Intergenic variant \\
\hline $6: 38360920$ & 0.87 & 1 & 82046 & Intergenic variant \\
\hline $6: 38399815$ & 0.86 & 0.93 & 120941 & Intergenic variant \\
\hline $6: 38441535$ & 0.8 & 0.93 & Intronic Variant LY49E \\
\hline
\end{tabular}

172 Table 4: Amino acid residue sequence in a conserved area of the 3'UTR found in all Equus

173 caballus LY49 genes as mapped in the EquCab2.0 assembly. The SNP position of

$1746: 38276955 \mathrm{G}>\mathrm{A}$ is highlighted in yellow.

\begin{tabular}{|l|l|}
\hline Gene & Sequence \\
\hline LY49B & AAAGACTTTCTCAGGGCCATTAAAGAGATGGGAAACTGCTTTCCAAAGAC \\
\hline LY49C & AGAGAATTTCCCAGGGCCATTAAAGAGAAGAGCAACTGATTTCCAAAGAC \\
\hline LY49D & AGAGAATTTCTCAGGGCCATTAAAGAGAAGGGCAACTGATTTCCAAAGAC \\
\hline LY49E & AGAGAATTTCTCAGGGCCATTAAAGAGAAGGGCAACTGATTTCCAAAGAC \\
\hline LY49F & AGAGAATTTTGCAGGGTCATTAAAGAGAGGGGTAACTGCTTTCCAAAGAC \\
\hline
\end{tabular}



(FPKM).

\begin{tabular}{|c|c|c|c|}
\hline Tissue & \multicolumn{3}{|c|}{ Gene count (FPKM) } \\
\hline \multirow{2}{*}{ Trophectoderm } & Day 15 & Day 22 & Day 25 \\
\cline { 2 - 4 } & 0.000 & 0.031 & 0.024 \\
\hline \multirow{2}{*}{ Inner cell mass } & Day 16 & Day 23 & Day 24 \\
\cline { 2 - 4 } & 0.00 & 0.00 & 0.00 \\
\hline
\end{tabular}

\section{Transcriptomic analysis of $R N A$ sequence data}

Measurable levels of $L Y 49 B$ mRNA were not detected in equine trophectoderm tissue collected on day 16 of development. However, LY49B mRNA was observed in trophectoderm tissue collected on days 23 and 24 of development (Table 5). Additionally, LY49B mRNA transcripts were detected in microarray data from equine chorion and chorionic girdle tissue between days 27 and 34 of development (Table S4). Inner cell mass tissue collected on days 15, 22 and 25 of development did not show any measurable transcription of LY49B (Table 5). The genotypes of the candidate SNPs in the mRNA samples analysed were unknown.

Table 5: Gene counts from RNA sequence data of three trophectoderm and three inner cell mass tissue samples from equine embryos. Transcript counts are in fragments per kilobase/million

\section{Discussion}

In this study, we aimed to identify variants at high frequencies in the Thoroughbred horse population that may be lethal in homozygous state. Analyses of genotype data from

190 Thoroughbred horses identified only two adjacent SNPs that fit the strict filtering criteria (Table

1). Genotype data from other horse breeds showed a similar reduction or absence of 
homozygotes for these SNPs (Table 2). Therefore, this two-SNP haplotype is a strong candidate for harbouring a variant that causes lethality in homozygous state.

\section{$L Y 49 B$ gene on ECA6. The $L Y 49 B$ gene belongs to the $L Y 49$ (Killer cell lectin-like receptor} subfamily A) family of receptors, which consists of five functional members in Equus caballus (49). Other species (including humans) have a functionally similar, but structurally different gene family called KIR (Killer cell immunoglobin line receptors) (50). The $L Y 49 / K I R$ gene family are expressed across various types of immune cells, and mediate their function through bindings to MHC-1 (51). The $L Y 49 B$ gene is expressed in myeloid cells where it regulates their activity through an inhibitory effect, possibly to prevent their spontaneous activation (52).

Despite the important role that they play in immunity, the function of $L Y 49$ genes in development is currently unknown. In humans, incompatibilities between foetal $K I R$ and maternal $M H C(H L A)$ genotypes are associated with an increased risk of miscarriage and preeclampsia (53-55). Additionally, knockdown of $L Y 49$ in mice showed a high rate of implantation failure $(56,57)$. These findings indicate that $L Y 49 B$ may play an important role in maternal/foetal compatibility and implantation success in horses.

Analysis of transcriptomic data found that $L Y 49 B$ was first expressed in equine trophoblast tissue during the placental development stage. The first evidence of $L Y 49 B$ expression was found on day 23-24 of development (Table 5), during which the glycoprotein capsule surrounding the embryo is broken down and placental tissue starts to develop (58). Measurable expression of $L Y 49 B$ was also found in chorion and chorionic girdle tissues between days 27 and 34 of development (Table S4). During this time, trophoblast cells rapidly proliferate to form the chorionic girdle, which then invades the endometrium to form epithelial cups (59). It 
215

216

217

218

219

220

221

222

223

224

225

226

227

228

229

230

231

232

233

234

235

236

is possible that $L Y 49 B$ is important for successful implantation of the embryo by mediating the action of MHC-1 which is expressed during this time $(60,61)$. However, it is also possible that loss of function in $L Y 49 B$ may result in post-natal juvenile death, which would explain the lack of homozygotes seen in our data. High rates of juvenile death would be more discernible in the population, so loss of function leading to embryonic death seems more likely. Further investigations into the role of $L Y 49 B$ in equine development would confirm whether impaired function causes lethality and the stage of development at which this occurs.

Variant calling in whole-genome sequence data from 90 domestic horses further confirmed an absence of minor homozygotes for the two SNPs of interest. Three variants closely linked to these SNPs were also identified in these data as the most likely candidates to cause loss of function in the $L Y 49 B$ gene and result in lethality in homozygous state (Table 3). One SNP was a missense mutation in the coding region of the $L Y 49 B$ gene that results in the substitution of a negatively charged serine for an aromatic phenylalanine residue. However, lack of conservation of this SNP in $L Y 49$ genes across taxa makes it seem unlikely to be a causative mutation for embryonic lethality. Two other variants found in the 3'UTR region of the $L Y 49 B$ gene were also closely linked to the SNPs identified in the preliminary analysis, and seemed more likely candidates to cause embryonic lethality in homozygous state.

The 3'UTR region of a gene is responsible for transcriptional stability through the binding of miRNAs and RNA binding proteins (62). The addition of the polyadenylation tail to the $3^{\prime} \mathrm{UTR}$ is also essential to ensure proper processing and translation of the mRNA strand (63). Mutations in the 3'UTR region can lead to degradation of the mRNA, resulting in reduced or inhibited translation even when the gene is transcribed (64). Variation in the $3^{\prime}$ UTR region of 
237

238

239

240

241

242

243

244

245

246

247

248

249

250 251 horses. $68)$.

genes are associated with a number of diseases including Huntington's and breast cancer in humans $(65,66)$. Additionally, SNPs in the 3'UTR region are associated with production traits in livestock including milk production in cows, muscularity in sheep and obesity in horses $(64,67$,

Despite the importance of the 3'UTR region for the mRNA stability and normal expression of a gene, little is known about how specific polymorphisms can affect posttranscriptional processing. This makes it difficult to identify how the 3'UTR variants identified in this study could affect the translation of $L Y 49 B$ mRNA into a functional protein. The 3'UTR variant 6:38276955G $>A$ was identified as a possible candidate for embryonic lethality because it is highly conserved between all members of the $L Y 49 B$ family (Table 4) in horses, so may play an important role in mRNA stability. The other 3'UTR variant $(6: 38276742 \mathrm{~A}>\mathrm{T})$ is found in an AU-rich region at the end of the transcript, so may be important in the addition of the polyadenylation tail. Further examination of the effects that these variants have on posttranscriptional processing would determine if they impact the normal expression of $L Y 49 B$ in

Despite an absence of homozygotes, the two intronic SNPs identified in this study were found at high heterozygote frequencies in the Thoroughbred population. Currently, there is no evidence that variation in the $L Y 49 B$ gene is associated with phenotypic advantages in horses. However, it is possible that one of the variants linked to these SNPs confers a heterozygote advantage, which could explain why they have reached such high frequencies in the breed. It is also possible that selective breeding practices favouring a limited number of stallion bloodlines are responsible for this potentially lethal haplotype drifting to high frequencies in the 
Thoroughbred population. This would be most likely to occur if a stallion that made a large genetic contribution to the population was a carrier. A similar instance has recently been documented in cattle, where a lethal mutation at high frequencies was traced back to a sire with an extensive genetic influence on a population (21).

The presence of this potentially lethal haplotype across many diverse breeds of domestic horses indicates that it may not be the result of a recent mutation present only in the

Thoroughbred population. Rather, heterozygotes for this haplotype may have been present in pre-domesticated horses as a rare variant, and have become more frequent in some domestic breeds as the result of population bottlenecks due to breed formations, selective breeding practices and potentially a heterozygote advantage. Domestication and breed formation events have been well documented to result in increased deleterious mutation loads in horses and other domestic species $(24,30,31,69)$. A high proportion of heterozygotes for this haplotype were found in some breeds closely related to the Thoroughbred including the Paint, French Trotter, Morgan and Quarter Horse. Notably, over 70\% of Quarter Horse samples included in this study were heterozygous for these SNPs. The Quarter Horse has an open stud book, and higher genetic diversity than the Thoroughbred population (46), making the high frequency of a potentially lethal haplotype at first surprising. The Quarter Horse dataset reportedly did not contain full or half siblings (46), but the collection of samples from one geographical area may not fully reflect the diversity of the worldwide population. An average relatedness analysis of these samples noted the large genetic influence of one particular Thoroughbred stallion (46), which may explain the high frequency of heterozygotes observed in this population. However, the extremely high frequency of heterozygotes in this breed may be due to a heterozygote advantage. 
The Belgian Draft, Mangalarga Paulista and Tuva breeds also show a high proportion of

282

283

284

285

286

287

288

289

290

291

292

293

294 heterozygotes, but are more distantly related to the Thoroughbred and to each other. Therefore, the high frequency of heterozygotes in these breeds may be due to independent genetic drift events. Heterozygotes for this haplotype were notably absent from one branch of the tree containing small heavy horses from Northern Europe, which are more distantly related to the

Thoroughbred. A larger dataset of Exmoor Pony samples from this phylogenetic branch revealed one heterozygote for this haplotype (Table 2). This could be due to a calling error, but it is also possible that these SNPs exist at very low frequencies in these breeds. The small sample size of the genotype data for many individual breeds in this study means that heterozygote frequencies across all subpopulations found throughout the world may deviate from that reported here. However, these data provide an indication of breeds with high proportions of heterozygotes for this region. Overall, our findings suggest that this region shows evidence harbouring a homozygous lethal variant, yet a high proportion of heterozygotes are found across many domestic horse breeds.

In this study, we identified a haplotype at high heterozygote frequencies in the Thoroughbred horse population that is a strong candidate for harbouring a variant causing lethality in homozygous state. Similar analyses on larger datasets in other livestock populations have identified multiple lethal haplotypes, so it is likely that other such variants are present at high frequencies in the Thoroughbred population but were not captured in this study. Additionally, the use of commercial SNP arrays only allows for the identification of variants with high minor allele frequencies in populations. Analysis of larger sample sizes, and using higher density genotype data could allow for identification of other variants associated with lethality in domestic horses. The identification of this potentially lethal haplotype demonstrates 
the potential implications of heavily favouring a limited number of bloodlines in selective breeding practices. The association of this haplotype with lethality can be used to assist in breeding decisions to improve mating outcomes in the Thoroughbred population. Although there are a high proportion of heterozygotes in some domestic breeds, limiting the use of stallions that are carriers could reduce the frequency of this haplotype in future generations. Additionally, further investigations into a possible heterozygote advantage could assist in understanding the high heterozygous frequencies across many domestic breeds. Further characterisation of lethal haplotypes in other breeds would also assist in breeding management to increase fertility rates in domestic horse populations.

\section{Methods}

\section{Initial genotyping}

Genotype data from a representative sample of Thoroughbreds were used to identify SNPs with a high proportion of heterozygotes, but an absence of homozygotes for one allele. Genome-wide SNP data were generated for 156 Australian Thoroughbred horses by genotyping samples on either the Illumina 70K Chip (65,102 SNPs) $(n=102)$ or the Affymetrix 670K Chip $(670,796$ SNPs) $(n=54)$. Common genotyped SNPs between the two arrays were scanned for deviations from the Hardy-Weinberg equilibrium with an absence of homozygotes for one allele using PLINK (version 1.9) (70). The $P$-values were adjusted using a false discovery rate correction with the R package "qvalue" (71). Since SNPs with an absence of homozygotes could indicate a calling error, the search was narrowed to only include adjacent SNPs that fit such criteria.

The frequencies of the candidate SNPs were then examined in publicly available genotype data from Japanese Thoroughbreds $(n=370)$ typed on the Affymetrix 670K Chip (43) 
and these were added the Thoroughbred sample. The SNP frequencies were then characterised from genotype data from Swedish Warmbloods $(n=380)$ (44) and Norwegian-Swedish Coldblooded Trotters $(n=670)$ (47) typed on the Affymetrix 670K Chip. Publicly available data from Exmoor Ponies ( $n=285$, typed on the Affymetrix 670K Chip) (47), Quarter Horses ( $n=$ 137, typed on the Illumina 70K Chip) (72) and horses of 32 different domestic breeds $(n=582$, typed on the Illumina 50K Chip) $(28,29)$ were also included in this preliminary scan for SNP frequencies. In these data, raw intensities were plotted to check for calling errors. If potential calling errors were detected, SNPs were recalled using a mixture model fitted with an expectation-maximization algorithm in $\mathrm{R}$.

\section{Variant discovery and mapping}

Publicly available whole-genome sequence data were used to further examine the frequencies of the candidate SNPs identified in the initial genotype analysis, and to identify linked variants. Paired end whole-genome sequence data from 90 horses of different domestic breeds were used in this analysis (Table S5). The whole genome datasets were downloaded from the European Nucleotide Archives (ENA, https://www.ebi.ac.uk/ena) which included horses of different domestic breeds (PRJEB14779, $n=70$ ) and additional Thoroughbred samples (PRJNA168142, $n=16$ and PRJNA184688, $n=4)$ (Table S5).

The SNP array used in the initial genotyping analysis was developed based on coordinates of the EquCab2.0 reference genome. For consistency, we used the EquCab2.0 assembly as a reference for the whole-genome sequence analysis. The raw reads were mapped to the EquCab2.0 reference genome using BWA-MEM algorithm from Burrows-Wheeler Alignment Tool (version 0.7.17) (73). Duplicate reads were flagged using Samblaster (version 
0.1.22) (74), and base recalibration was performed using Genome Analysis Toolkit (GATK)

(version 4.0.8.1) (75). Variants (SNPs and INDELs (insertions and deletions)) were called using

(76). The individual SNPs were then filtered to only include high quality allele calls with an genes of interest in embryonic tissue. The data included equine inner cell mass tissue (collected the Functional Annotation of ANimal Genomes (FAANG) equine biobank (available from ENA under the project name PRJNA223157) (78). Adaptors were trimmed using bbduk from BBtools (version 37.98) (79). Reads were aligned to the EquCab 2.0 genome using STAR (version 2.7.2b) (80). Counts were generated using featurecounts from subread (version 1.5.1) (81), then quantified in fragments per kilobase/million (FPKM) using the R package "edgeR" (82) with the

367 Equus_caballus_Ensembl_94 file used for annotation. Microarray data for chorion $(n=19)$ and 368 chorionic girdle $(n=19)$ tissue collected from horse embryos between days 27-34 of 369 development (83) were also examined for gene expression levels. 
DNA samples for Australian Thoroughbred horses were collected under approval from University of Sydney Ethics Committee N00-2009-3-5109. Written informed consent to use the

373 animals in this study was obtained from the owners of the animals. The hair samples from

374 Swedish Warmblood horses were originally collected for parentage testing and stored in the

375 biobank at the Animal Genetics Laboratory, SLU so ethics approval was not applicable. DNA

376 samples of Norwegian-Swedish Coldblooded Trotter were collected under approval from the

377 Ethics Committee for Animal Experiments in Uppsala, Sweden [Number: C 121/14]. All other

378 data was downloaded from publicly available repositories.

379 Acknowledgements

380 The authors acknowledge the technical assistance provided by the Sydney Informatics Hub, a 381 Core Research Facility of the University of Sydney.

382 Figure 1: The equine $L Y 49 B$ gene structure and SNP positions. The two variants in the 383 intronic region (6:38278097 and 6:38278874) were identified in preliminary analysis as showing 384 a significant absence of homozygotes for one allele. The three variants marked with a $*$ are in 385 linkage disequilibrium to these SNPs and may cause a loss of function in homozygous state. The 386 structure of the gene is based on the EquCab 2.0 reference genome where the $L Y 49 B$ gene is on 387 the reverse strand

388 Supporting Information: Additional tables and figures. 


\section{References}

392 1. Spencer TE. Early pregnancy: concepts, challenges, and potential solutions. Anim Front. $393 \quad 2013 ; 3(4): 48-55$.

394 2. White JK, Gerdin AK, Karp Natasha A, Ryder E, Buljan M, Bussell James N, et al.

395 Genome-wide generation and systematic phenotyping of knockout mice reveals new roles for 396 many genes. Cell. 2013;154(2):452-64.

397 3. Mary ED, Ann MF, Xiao J, Lydia T, Michael DW, Jacqueline KW, et al. High398 throughput discovery of novel developmental phenotypes. Nature. 2016;537(7621).

399 4. Gao Z, Waggoner D, Stephens M, Ober C, Przeworski M. An estimate of the average 400 number of recessive lethal mutations carried by humans. Genetics. 2015;199(4):1243-54.

401 5. Ballinger MA, Noor MAF. Are lethal alleles too abundant in humans? Trends Genet. $402 \quad 2018 ; 34(2): 87-9$.

403 6. Mukai T, Chigusa SI, Mettler LE, Crow JF. Mutation rate and dominance of genes 404 affecting viability in Drosophila melanogaster. Genetics. 1972;72(2):335.

405 7. Sulem P, Helgason H, Oddson A, Stefansson H, Gudjonsson SA, Zink F, et al.

406 Identification of a large set of rare complete human knockouts. Nat Genet. 2015;47(5):448-52.

407 8. Curik I, Ferencakovic M, Solkner J. Inbreeding and runs of homozygosity: a possible 408 solution to an old problem. Livest Sci. 2014;166(1):26-34.

409 9. Keller LF, Waller DM. Inbreeding effects in wild populations. Trends Ecol Evol. $410 \quad 2002 ; 17(5): 230-41$.

411 10. Charlesworth D, Willis JH. The genetics of inbreeding depression. Nat Rev Genet. $412 \quad 2009 ; 10(11): 783-96$. 
413 11. Trask AE, Bignal EM, McCracken DI, Monaghan P, Piertney SB, Reid JM. Evidence of 414 the phenotypic expression of a lethal recessive allele under inbreeding in a wild population of 415 conservation concern. J Anim Ecol. 2016;85(4):879-91.

416 12. Bosse M, Derks M, Groenen M. Deleterious alleles in the context of domestication, 417 inbreeding, and selection. Evol Appl. 2019;12(1):6-17.

418 13. Hoff JL, Decker JE, Schnabel RD, Taylor JF. Candidate lethal haplotypes and causal 419 mutations in Angus cattle. BMC Genomics. 2017;18(1).

420 14. VanRaden PM, Olson KM, Null DJ, Hutchison JL. Harmful recessive effects on fertility 421 detected by absence of homozygous haplotypes. J Dairy Sci. 2011;94(12):6153-61.

422 15. Fasquelle C, Sartelet A, Li W, Dive M, Tamma N, Michaux C, et al. Balancing selection 423 of a frame-shift mutation in the MRC2 gene accounts for the outbreak of the crooked tail 424 syndrome in Belgian Blue cattle. PLoS Genet. 2009;5(9):e1000666.

425 16. Zhang C, MacNeil MD, Kemp RA, Dyck MK, Plastow GS. Putative loci causing early 426 embryonic mortality in Duroc swine. Front Genet. 2018;17(9):655.

427 17. Charlier C, Li W, Harland C, Littlejohn M, Coppieters W, Creagh F, et al. NGS-based 428 reverse genetic screen for common embryonic lethal mutations compromising fertility in 429 livestock. Genome Res. 2016;26(10):1333-41.

430 18. Sonstegard TS, Cole JB, VanRaden PM, van Tassell CP, Null DJ, Schroeder SG, et al. 431 Identification of a nonsense mutation in CWC15 associated with decreased reproductive 432 efficiency in Jersey cattle. PLOS ONE. 2013;8(1):e54872.

433 19. Derks MFL, Megens HJ, Bosse M, Lopes MS, Harlizius B, Groenen MAM. A systematic 434 survey to identify lethal recessive variation in highly managed pig populations. BMC Genomics. $435 \quad 2017 ; 18(1)$. 
436 20. Derks MFL, Gjuvsland AB, Bosse M, Lopes MS, van Son M, Harlizius B, et al. Loss of

437 function mutations in essential genes cause embryonic lethality in pigs. PLoS Genet.

$438 \quad 2019 ; 15(3): \mathrm{e} 1008055$.

439 21. Bourneuf E, Otz P, Pausch H, Jagannathan V, Michot P, Grohs C, et al. Rapid discovery

440 of de novo deleterious mutations in cattle enhances the value of livestock as model species. Sci

$441 \quad$ Rep. 2017;7(1):11466.

442 22. Schubert M, Jónsson H, Chang D, Sarkissian CD, Ermini L, Ginolhac A, et al. Prehistoric 443 genomes reveal the genetic foundation and cost of horse domestication. Proc Natl Acad Sci U S

A. 2014;111(52):E5661-E9.

445 23. Cruz F, Vilà C, Webster MT, Medicinska och farmaceutiska v, Medicinska f,

446 Evolutionsbiologi, et al. The legacy of domestication: accumulation of deleterious mutations in

447 the dog genome. Mol Biol Evol. 2008;25(11):2331-6.

448 24. Marsden CD, Vecchyo DO-D, O’Brien DP, Taylor JF, Ramirez O, Vilà C, et al.

449 Bottlenecks and selective sweeps during domestication have increased deleterious genetic

450 variation in dogs. Proc Natl Acad Sci U S A. 2016;113(1):152-7.

451 25. García-Dorado A. Understanding and predicting the fitness decline of shrunk

452 populations: Inbreeding, purging, mutation, and standard selection. Genetics. 2012;190(4):1461-

45376.

454 26. Casas E, Kehrli ME. A review of selected genes with known effects on performance and

455 health of cattle. Front Vet Sci. 2016;3.

456 27. Jagannathan V, Gerber V, Rieder S, Tetens J, Thaller G, Drögemüller C, et al.

457 Comprehensive characterization of horse genome variation by whole-genome sequencing of 88

458 horses. Anim Genet. 2019;50(1):74-7. 
459 28. Petersen JL, Mickelson JR, Rendahl AK, Valberg SJ, Andersson LS, Axelsson J, et al.

460 Genome-wide analysis reveals selection for important traits in domestic horse breeds. PLoS

461 Genet. 2013;9(1):e1003211.

462 29. Petersen JL, Mickelson JR, Cothran EG, Andersson LS, Axelsson J, Bailey E, et al.

463 Genetic diversity in the modern horse illustrated from genome-wide SNP data. PLOS ONE.

$464 \quad 2013 ; 8(1): \mathrm{e} 54997$.

465 30. Orlando L, Librado P. Origin and evolution of deleterious mutations in horses. Genes.

$466 \quad 2019 ; 10(9): 649$

467 31. Fages A, Hanghøj K, Khan N, Gaunitz C, Seguin-Orlando A, Leonardi M, et al. Tracking

468 five millennia of horse management with extensive ancient genome time series. Cell.

$469 \quad 2019 ; 177(6): 1419-35 . e 31$.

470 32. Librado P, Fages A, Gaunitz C, Leonardi M, Wagner S, Khan N, et al. The evolutionary

471 origin and genetic makeup of domestic horses. Genetics. 2016;204(2):423-34

472 33. Aurich C. Reproductive cycles of horses. Anim Reprod Sci. 2011;124(3):220-8.

473 34. Allen WR, Wilsher S. Half a century of equine reproduction research and application:

474 A veterinary tour de force. Equine Vet J. 2018;50(1):10-21.

475 35. Weatherby and Sons. An Introduction to the General Stud Book. London: Weatherby and 476 Sons; 1791.

477 36. Hill EW, Gu J, McGivney BA, MacHugh DE. Targets of selection in the Thoroughbred

478 genome contain exercise-relevant gene SNPs associated with elite racecourse performance.

479 Anim Genet. 2010;41(2):56-63.

480 37. Gu J, Orr N, Park SD, Katz LM, Sulimova G, MacHugh DE, et al. A genome scan for

481 positive selection in Thoroughbred horses. PLOS ONE. 2009;4(6):e5767. 
482 38. Cunningham EP, Dooley JJ, Splan RK, Bradley DG. Microsatellite diversity, pedigree

483 relatedness and the contributions of founder lineages to Throughbred horses. Anim Genet.

$484 \quad 2001 ; 32(6): 360$.

485 39. Todd ET, Ho SYW, Thomson PC, Ang RA, Velie BD, Hamilton NA. Founder-specific

486 inbreeding depression affects racing performance in Thoroughbred horses. Sci Rep.

$487 \quad 2018 ; 8(1): 6167$.

488 40. Corbin LJ, Blott SC, Swinburne JE, Vaudin M, Bishop SC, Woolliams JA. Linkage

489 disequilibrium and historical effective population size in the Thoroughbred horse. Anim Genet.

$490 \quad 2010 ; 41(2): 8-15$.

491 41. McGivney BA, Han H, Corduff LR, Katz LM, Tozaki T, MacHugh DE, et al. Genomic

492 inbreeding trends, influential sire lines and selection in the global Thoroughbred horse

493 population. Sci Rep. 2020;10(1):466.

494 42. Woolliams JA, Berg P, Dagnachew BS, Meuwissen THE. Genetic contributions and their 495 optimization. J Anim Breed Genet. 2015;132(2):89-99.

496 43. Fawcett JA, Sato F, Sakamoto T, Iwasaki WM, Tozaki T, Innan H, et al. Genome-wide 497 SNP analysis of Japanese Thoroughbred racehorses. PLOS ONE. 2019;14(7).

498 44. Ng PC, Henikoff S. SIFT: Predicting amino acid changes that affect protein function.

499 Nucleic Acids Res. 2003;31(13):3812-4.

500 45. Futas J, Horin P. Natural killer cell receptor genes in the family Equidae: not only Ly49.

$501 \quad$ PLOS ONE. 2013;8(5):e64736.

502 46. Kelley J, Walter L, Trowsdale J. Comparative genomics of natural killer cell receptor

503 gene clusters. PLoS Genet. 2005;1(2):129-39. 
47. Rahim MMA, Tu MM, Mahmoud AB, Wight A, Abou-Samra E, Lima PDA, et al. Ly49

505

506

507

508

509

510

511

512

513

514

515

516

517 52. Leon L, Felker AM, Kay VR, Tu MM, Makrigiannis AP, Croy BA. Ly49 knockdown in

518 mice results in aberrant uterine crypt formation and impaired blastocyst implantation. Placenta.

$519 \quad 2016 ; 39: 147-50$.

520 53. Lima PD, Tu M, M., Rahim MM, A., Peng A, R. Peng, Croww AC, Makrigiannis A, P.

521 . Ly49 receptors activate angiogenic mouse DBA+ uterine natural killer cells. Cellular and

522 Molecular Immunology. 2014;11(5):467-76.

523 54. Allen WR, Wilsher S. A review of implantation and early placentation in the mare.

524

49. Hiby S, Regan L, Lo W, Farrell L, Carrington M, Moffett A. Association of maternal

killer-cell immunoglobulin-like receptors and parental HLA-C genotypes with recurrent miscarriage. Hum Reprod. 2008;23(4):972-6.

50. Hiby SE, Apps R, Sharkey AM, Farrell LE, Gardner L, Mulder A, et al. Materal activating KIRs protect against human reproductive failure mediated by fetal HLA-C2. J Clin Investig. 2010;120(11):4102.

51. Long W, Shi Z, Fan S, Liu L, Lu Y, Guo X, et al. Association of maternal KIR and fetal HLA-C genes with the risk of preeclampsia in the Chinese Han population. Placenta. 2015;36(4):433-7.

Placenta. 2009;30(12):1005-15. 
525 55. Noronha LE, Antczak DF. Maternal Immune Responses to Trophoblast: The

526 Contribution of the Horse to Pregnancy Immunology. Am J Reprod Immunol. 2010;64(4):231-

52744

528 56. Donaldson WL, Oriol JG, Pelkaus CL, Antczak DF. Paternal and maternal major

529 histocompatibility complex class I antigens are expressed co-dominantly by equine trophoblast.

530 Placenta. 1994;15(2):123-35.

531 57. Bacon SJ, Ellis SA, Antczak DF. Control of expression of major histocompatibility

532 complex genes in horse trophoblast. Biol Reprod. 2002;66(6):1612-20.

533 58. Steri M, Idda ML, Whalen MB, Orrù V. Genetic variants in mRNA untranslated regions.

534 Wiley Interdiscip Rev RNA. 2018;9(4):n/a-n/a.

535 59. Di Giammartino DC, Nishida K, Manley James 1. Mechanisms and consequences of 536 alternative polyadenylation. Mol Cell. 2011;43(6):853-66.

537 60. Lewis SL, Holl HM, Streeter C, Posbergh C, Schanbacher BJ, Place NJ, et al.

538 Genomewide association study reveals a risk locus for equine metabolic syndrome in the

539 Arabian horse 1. J Anim Sci. 2017;95(3):1071-9.

540 61. Dorairaj J, Salzman D, Wall D, Rounds T, Preskill C, Sullivan C, et al. A germline

541 mutation in the BRCA1 3'UTR predicts Stage IV breast cancer. BMC Cancer. 2014;14(1):421.

542 62. Kim KH, Keum J, Seong D, Kwak S, Lee R, Gusella J, et al. Full sequence of mutant

543 huntingtin 3 '-untranslated region and modulation of its gene regulatory activity by endogenous

544 microRNA. J Hum Genet. 2019;64(10):995-1004.

545 63. Hou J, An X, Song Y, Gao T, Lei Y, Cao B. Two mutations in the caprine MTHFR

546 3'UTR Regulated by microRNAs are associated with milk production traits. PLOS ONE.

$547 \quad 2015 ; 10(7): \mathrm{e} 0133015-\mathrm{e}$. 
548 64. Alex C, Fabienne M, Haruko T, Dimitri P, Xavier T, Bernard B, et al. A mutation

549 creating a potential illegitimate microRNA target site in the myostatin gene affects muscularity

550 in sheep. Nat Genet. 2006;38(7):813.

551 65. Librado P, Gamba C, Gaunitz C, Der Sarkissian C, Pruvost M, Albrechtsen A, et al.

552 Ancient genomic changes associated with domestication of the horse. Science.

$553 \quad 2017 ; 356(6336): 442-5$.

554 66. Petersen JL, Mickelson JR, Cleary KD, McCue ME. The American Quarter Horse:

555 population structure and relationship to the Thoroughbred. J Hered. 2014;105(2):148-62.

556 67. Purcell S, Neale B, Todd-Brown K, Thomas L, Ferreira MAR, Bender D, et al. PLINK:

557 A tool set for whole-genome association and population-based linkage analyses. Am J Hum

558 Genet. 2007;81(3):559-75.

559 68. Storey J, Bass A, Dabney A, Robinson D. qvalue: Q-value estimation for false discovery

560 rate control. R package version 2.18.0 ed: http://github.com/jdstorey/qvalue; 2019.

561 69. Ablondi M, Viklund A, Lindgren G, Eriksson S, Mikko S. Signatures of selection in the

562 genome of Swedish Warmblood horses selected for sport performance. BMC Genomics.

$563 \quad 2019 ; 20(1)$.

564 70. Velie BD, Shrestha M, François L, Schurink A, Tesfayonas YG, Stinckens A, et al. Using

565 an inbred horse breed in a high density genome-wide scan for genetic risk factors of insect bite

566 hypersensitivity (IBH). PLOS ONE. 2016;11(4).

567 71. Petersen JL, Valberg SJ, Mickelson JR, McCue ME. Haplotype diversity in the equine 568 myostatin gene with focus on variants associated with race distance propensity and muscle fibre 569 type proportions. Anim Genet. 2014;45(6):827-35. 
570 72. Li H, Durbin R. Fast and accurate short read alignment with Burrows-Wheeler

571 transform. Bioinformatics. 2009;25(14):1754-60.

572 73. Faust G, Hall I. SAMBLASTER: fast duplicate marking and structural variant read

573 extraction. Bioinformatics. 2014;30(17):2503-5.

574 74. McKenna A, Hanna M, Banks E, Sivachenko A, Cibulskis K, Kernytsky A, et al. The

575 Genome Analysis Toolkit: A MapReduce framework for analyzing next-generation DNA

576 sequencing data.(Report). Genome Res. 2010;20(9):1297-303.

577 75. Depristo MA, Banks E, Poplin R, Garimella KV, Maguire JR, Hartl C, et al. A

578 framework for variation discovery and genotyping using next-generation DNA sequencing data.

$579 \quad$ Nat Genet. 2011;43(5):491.

580 76. Lu S, Wang J, Chitsaz F, Derbyshire MK, Geer RC, Gonzales NR, et al.

581 CDD/SPARCLE: the conserved domain database in 2020. Nucleic Acids Res. 2019;48.

582 77. Iqbal K, Chitwood JL, Meyers-Brown GA, Roser JF, Ross PJ. RNA-Seq transcriptome 583 profiling of equine inner cell mass and trophectoderm. Biol Reprod. 2014;90(3).

584 78. Bushnell B. BBtools sourceforge.net/projects/bbmap/ 2019 [

585 79. Dobin A, Davis CA, Schlesinger F, Drenkow J, Zaleski C, Jha S, et al. STAR: ultrafast 586 universal RNA-seq aligner. Bioinformatics. 2013;29(1):15-21.

587 80. Liao Y, Smyth GK, Shi W. FeatureCounts: an efficient general purpose program for 588 assigning sequence reads to genomic features. Bioinformatics. 2014;30(7):923-30.

589 81. Robinson MD, McCarthy DJ, Smyth GK. edgeR : a Bioconductor package for 590 differential expression analysis of digital gene expression data. Bioinformatics. 2010;26(1):139-

59140 
592 82. Read JE, Cabrera-Sharp V, Offord V, Mirczuk SM, Allen SP, Fowkes RC, et al.

593 Dynamic changes in gene expression and signalling during trophoblast development in the horse.

594 Reproduction. 2018;156(4).

595 83. Velie BD, Jäderkvist Fegraeus K, Solé M, Rosengren MK, Røed KH, Ihler C-F, et al. A

596 genome-wide association study for harness racing success in the Norwegian-Swedish

597 coldblooded trotter reveals genes for learning and energy metabolism. BMC Genet.

$598 \quad 2018 ; 19(1): 1-13$. 


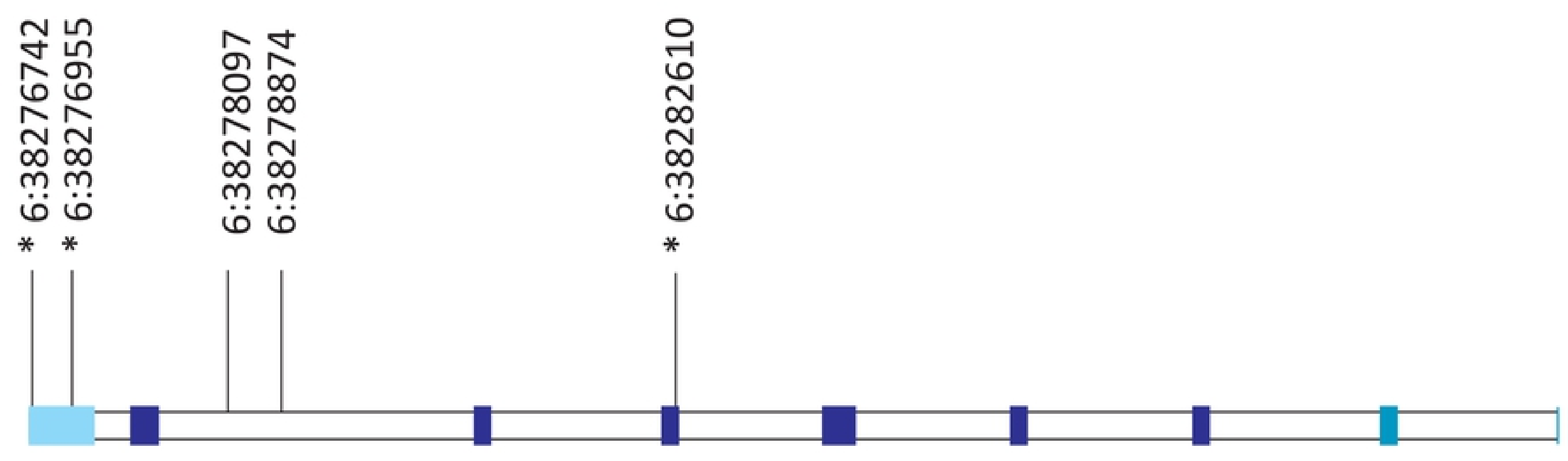

14,204 bases

exonic region

3'UTR region

5'UTR region

intronic region

Figure 1 